\title{
Minimally Invasive Approach to Supra-pubic and Non-Midline Lower Abdominal Ventral Hernia - An Extended Indication of TAPE Technique
}

\author{
Joe King Man FAN, MBBS MS (HKU) FRCSEd'², Jeremy YIP, MBBS FRCSEd'1 Matrix Fung, MBBS MRCSEd¹, Oswens Siu Hung LO,

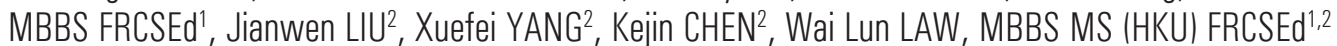 \\ 'Department of Surgery, The University of Hong Kong, Li Ka Shing Faculty of Medicine, HKSAR, ${ }^{2}$ Department of Surgery, The University of Hong Kong- \\ Shenzhen Hospital, Shenzhen, China
}

\begin{abstract}
Repair of lower abdominal incisional hernia is always a surgical challenge. TAPE technique has been described for the repair of supra-pubic midline incisional hernia with satisfactory outcome. Its indication can be extended for treatment of non-midline lower abdominal hernia. Peritoneal incision is created just below the hernia defect with pre-peritoneal dissection to expose supra-pubic preperitoneal space with Cooper's ligament exposed. Non-adhesive mesh then placed over preperitoneal space and partially intra-peritoneally, and cover the whole extra-peritoneal space prepared to ensure enough overlapping. Mesh is fixed by tackers for intra-peritoneal part, most inferior fixation points were at peritoneal incision line. Extra-peritoneal part of meshes is fixed at the safety zone and covered up by the peritoneal flap to avoid mesh migration. Fixation of the meshes at the lateral aspects were facilitated by the peritoneal flap and subsequent fibrosis and adhesion to the extra-peritoneal structures in cases of lateral lower abdominal hernia. Repair of midline and lateral lower abdominal incisional hernia with this novel modified technique with prosthetic mesh is safe and effective. A larger case series and longer follow-up is required for validation of this technique.
\end{abstract}

Keywords: Supra-pubic, Non-midline Ventral Hernia, Ventral Hernia, TAPE, Laparoscopic
Received August 4, 2017

Accepted August 9, 2017

Corresponding author

Joe King Man FAN

Department of Surgery, Li Ka Shing Faculty of Medicine, The University of Hong Kong, Pokfulam Road,

HKSAR, China

Tel: $+852-22554763$

Fax: +852-28728425

E-mail: drjoefan@hku.hk

Part of the data in manuscript has been presented in 1st World Conference on Abdominal Wall Hernia Surgery, Milan, Italy, Apr 2015.

Copyright (๑) 2017 The Journal of Minimally Invasive Surgery. All rights reserved.

\section{INTRODUCTION}

Laparoscopic ventral hernia repair has been proven to be superior to open approach with less hernia recurrence, better post-operative pain control and minimize wound related complications. ${ }^{1,2}$ On the other hand, its advantages subject to good case selections and surgeons' experience. With the intraperitoneal onlay mesh (IPOM) approach, meshes with nonadhesive surface in one side were placed intra-peritoneally and subsequent fixation by trans-abdominal sutures and tackers for the completion of repair is superior to suture repair alone. ${ }^{3}$ Recent evidences suggest that mesh augmentation technique results in lesser recurrence than bridging technique. ${ }^{4}$ Success of the surgery depends on adequacy of adhesiolysis, coverage of the defect by prosthetic meshes and secureness of fixation.

In which, there are certain areas that are prone to develop post-repair recurrence, these are areas where meshes may not 
be securely fixed at its desired position or inadequate overlapping of defect due to anatomical considerations. In particular, supra-pubic or lower abdominal hernia are prone to recur after hernioplasty due to: 1 . Highest pressure at lower abdominal wall at erect position, open suturing repair will become a tension repair if patient at upright position; 2 . This is where important structures are, including urinary bladder, iliac vessels, etc. This makes mobilization of healthy fascial layers for apposition difficult and impossible for fixation of mesh at these areas with conventional laparoscopic approach - which ends up in inadequate coverage and fixation and finally hernia recurrence.

Laparoscopic repair of supra-pubic hernia has been described in 2000s by Carbonell et al., ${ }^{5}$ Palanivelu et al. ${ }^{6}$ and Varnell et al. ${ }^{7}$ Complication rate ranged from $16.6 \%$ to $38 \%$ and overall recurrence of around $6 \%$. The concept of laparoscopic trans-abdominal partial extra-peritoneal (TAPE) technique was further consolidated by Sharma et al. and published in 2011. ${ }^{8}$ With this hybrid approach, part of the prosthetic mesh is being placed intra-peritoneally to cover the defect, where the inferior part is being placed at pre-peritoneal space and covered up by the peritoneal flap after completion of procedure (Fig. 1). And the intra-peritoneal part will be same as usual IPOM repair, which can solve the problem of adequacy of mesh coverage and fixation. TAPE technique was originally described for application in patients with supra-pubic hernia, for those with lateral extension, or originated from lateral

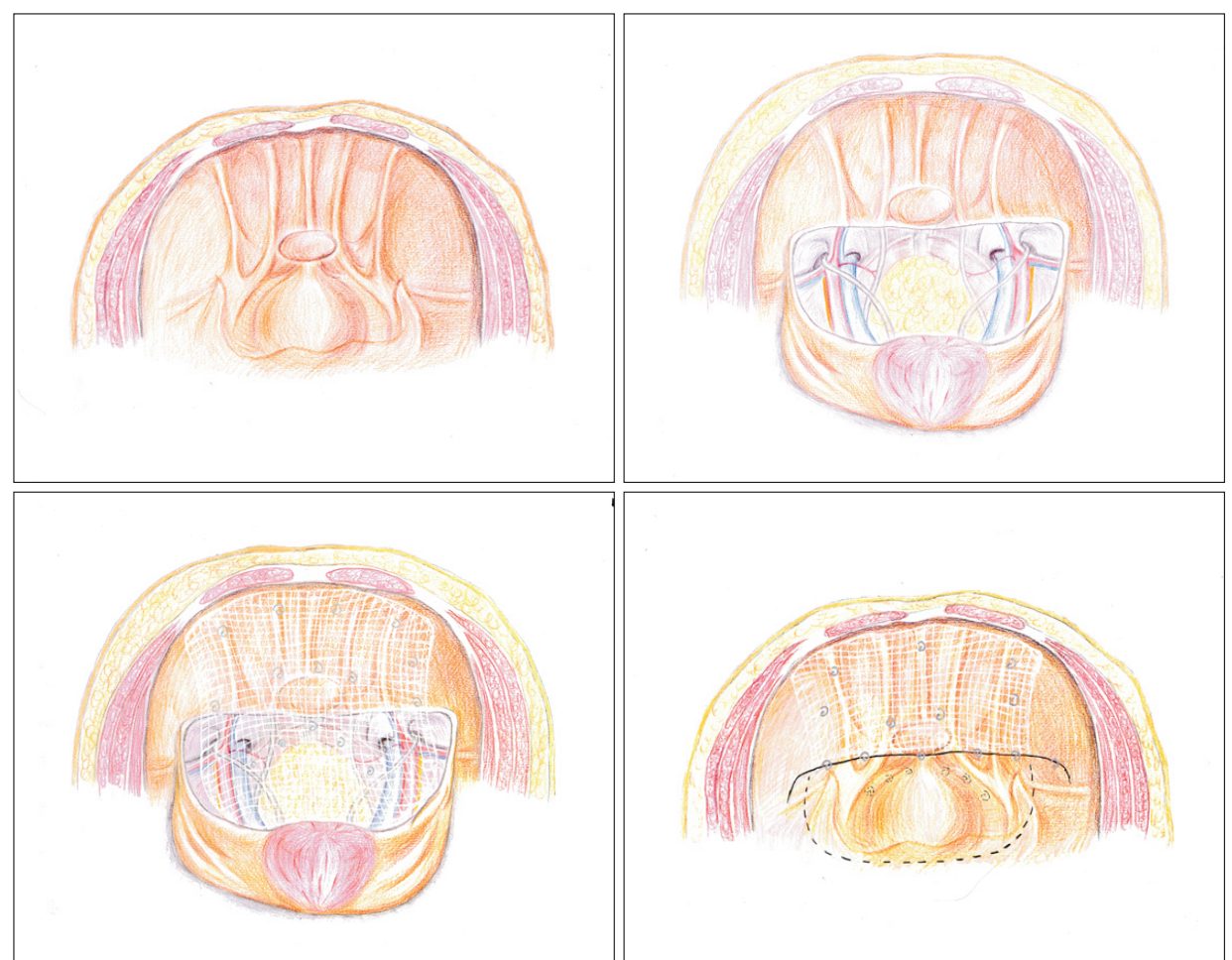

Fig. 1. Original TAPE technique described by Sharma A et al. lower abdominal wall, we need a special design of surgical approach to solve the problem of mesh fixation and defect coverage at this special region where it's dangerous for us to fix the mesh on. There were limited reports or experience on this distinct clinical entity. ${ }^{9,10}$ In view of clinical difficulties in this aspect, a novel modification of the TAPE technique was studied and explored in our center for patients with midline and lateral lower abdominal hernia.

\section{METHOD}

\section{Operative details}

All operations were performed under general anesthesia at supine positions. Urinary catheters were routinely inserted for decompression. Prophylactic antibiotic was not routinely given. First trocar was inserted at left upper quadrant or side opposite to the defect by open technique (e.g. trocar was inserted at left abdominal wall for repair of right paramedian hernia), in case for repair of post-TRAM incisional hernia, a supra-umbilical incision was made for insertion of trocar. Followed by insertion of three to four $5 \mathrm{~mm}$ trocars for adhesiolysis, preperitoneal dissection, placement and fixation of mesh. Here is the author's recommended method for performing midline and non-midline lower abdominal ventral hernia with extended indication of TAPE technique. 


\section{Peritoneal incision}

Peritoneal incision is started from medial side, immediate above the dome of bladder and extend to lateral aspect with monopolar diathermy hook. The merit of using monopolar diathermy for the initial dissection is it can achieve a sharp and neat cut on the peritoneum. This is an important issue as ultrasonic dissector will cause shrinkage of peritoneum or tissue loss, this will affect subsequent quality and coverage of peritoneal flap after the placement of prosthetic mesh. When it came to where the defect is, the incision should be kept just inferior to the hernia defect and extend laterally. The peritoneal dissection should be all the way to level of anterior superior iliac spine or preferably level of umbilicus. If the defect extend to midline or coverage of defect by mesh is less than $5 \mathrm{~cm}$, the peritoneal incision at the midline should be extended to the contralateral side for a comfortable and nice accommodation of mesh without any folding.

\section{Pre-peritoneal dissection}

The pre-peritoneal dissection was similar to TAPP - the peritoneal flap is raised at least $4 \sim 5 \mathrm{~cm}$ away from deep inguinal ring, with triangle of Doom, vas deference, round ligament and gonadal vessels skeletonized and exposed. Where at the lateral aspect, dissection at pre-peritoneal level with protection of ilioinguinal nerves and lateral cutaneous nerves of thigh (Triangle of pain). Pre-vesicle space is recommended to be prepared with urinary bladder fully mobilized and bilateral Coopers' ligaments exposed for secured mesh placement and fixation. This is to ensure there will be no folding of extraperitoneal part of the mesh. The larger the pre-peritoneal space prepared, the easier the mesh placement. However, unnecessary pre-peritoneal dissection without mesh coverage will potentially weaken anterior abdominal wall and end up in occurrence of hernia.

\section{Mesh positioning and fixation}

The PARIETEX COMPOSITE ${ }^{\circledR}$ (Medtronics $^{\mathrm{TM}}$ ) mesh was used in our series. The position of the mesh should be estimated and marked on the skin of abdominal wall. Unlike normal IPOM repair, where all 4 sutures on the edges of the mesh can be retrieved to skin level for fixation, only the superior and both lateral transabdominal sutures (TAS) could be brought to cutaneous layer in repair of lower abdominal hernia. The mesh should be fixed by using either titanium or absorbable tackers from cranial to caudal manner and end at the level of initial peritoneal incision line. Additional row of tacks should be fired along this line as this serves as most inferior point of fixation before the "dangerous zone. After that, the mesh can be further fixed at the anterior abdominal wall in front of the urinary bladder, and Cooper's ligament that is similar to TAPP repair for inguinal hernia at the medial aspect but not beyond the triangle of doom and triangle of pain laterally. The mesh is now preliminary fixed at the pre-

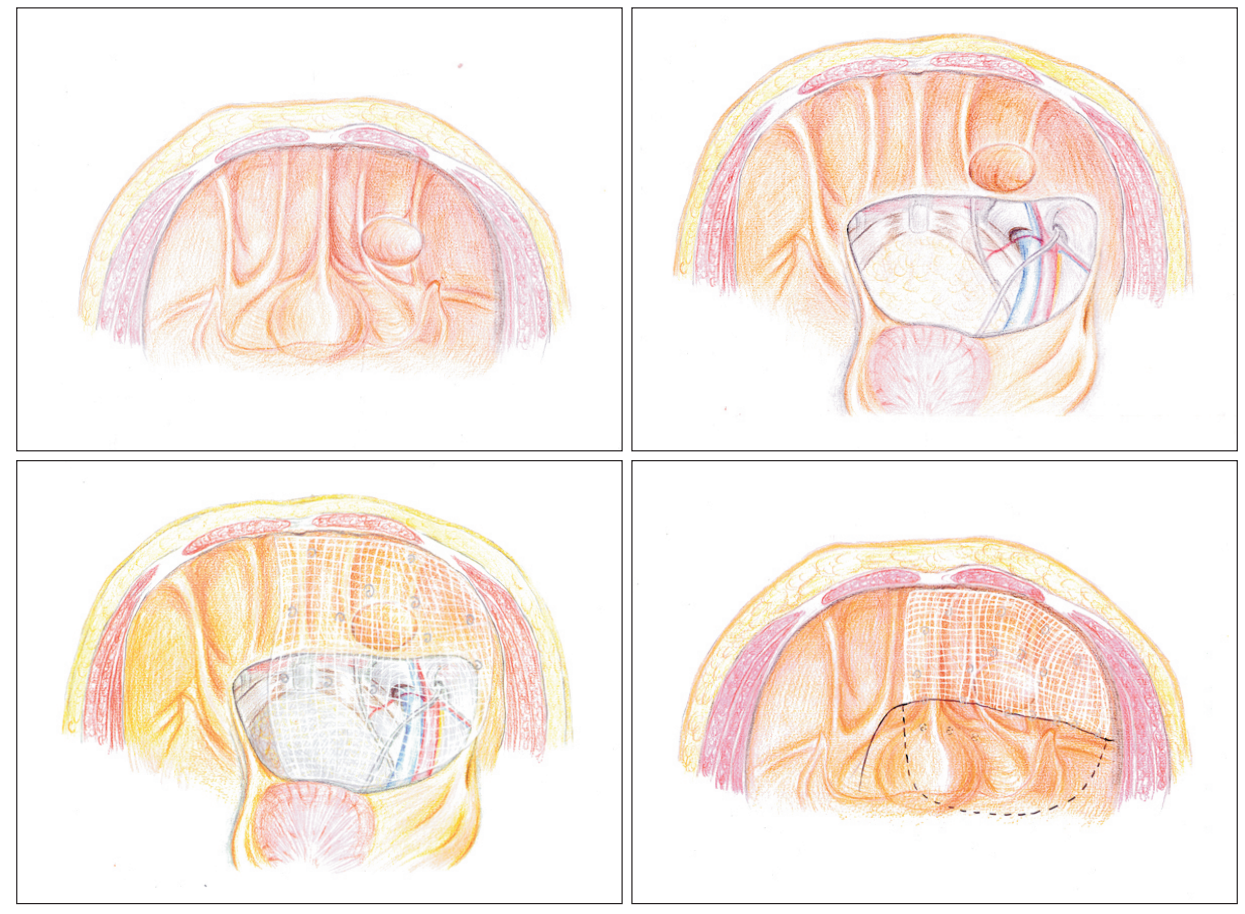

Fig. 2. Extended indication for lower lateral abdominal hernia, where the composite mesh over inferior lateral aspect (triangle of Doom and triangle of pain) is only fixed by overlaying peritoneal flap repositioned. 
peritoneal space and on the abdominal wall above the peritoneal incision line, then the urinary bladder and peritoneal flap is fixed to its original position/dissection point by tackers. One important point to noted was no tacker should be fired below this peritoneal edge on the peritoneal side at this stage because pre-peritoneal structures were not well visualized after peritoneal flap was reposition to its original position, any tacking or fixation is extremely dangerous and may potentially injure the iliac vessels or nerves. Finally, the double-crowning fixation was completed with additional tacks on the abdominal wall above the peritoneal incision line (Fig. 2).

Table 1. Different types of midline hernia

\begin{tabular}{|lcc|}
\hline & Non-suprapubic hernia (n) & Suprapubic hernia \\
\hline Incisional & 41 & 9 \\
\hline Para-umbilical & 31 & - \\
\hline Umbilical & 7 & - \\
\hline Epigastric & 3 & - \\
Spigelian & 2 & - \\
\hline Mixed & 5 & - \\
\hline Subtotal & 89 & 9 \\
\hline
\end{tabular}

\section{RESULTS AND OUR CENTRE EXPERIENCE}

\section{For midline suprapubic hernia}

IPOM operations performed in all the affiliated hospitals of the University of Hong Kong (Queen Mary Hospital, HKSAR, China; Tung Wah Hospital, HKSAR, China; The University of Hong Kong - Shenzhen Hospital, Shenzhen, China) between January 2008 and December 2014 were reviewed. Patients' demographics, operative records, postoperative morbidities and recurrence rates were retrieved from the electronic medical records retrospectively. Suprapubic hernias were defined when the inferior margin of the hernia was within $5 \mathrm{~cm}$ from the pubic bone. Other midline ventral hernias were defined as non-suprapubic hernias.

Table 2. Previous operations and incisions of patients with suprapubic hernia

\begin{tabular}{lc|}
\hline \multicolumn{1}{|c}{ Previous operation and incision } & Number of suprapubic hernia \\
\hline Caesarean section/lower midline & 3 \\
\hline TAHBSO/lower midline & 1 \\
\hline Female sterilization/lower midline & 1 \\
\hline Low anterior resection/lower midline & 1 \\
\hline AAA open repair/midline & 1 \\
\hline Exploratory laparotomy/midline & 2 \\
\hline
\end{tabular}

Table 3. Comparing the surgical outcomes of suprapubic vs non-suprapubic ventral hernia repair

\begin{tabular}{|lccc|}
\hline & Non-suprapubic hernia $(\mathbf{n}=89)$ & Suprapubic hernia $(\mathbf{n}=9)$ & $p$ value \\
\hline Mean age & 65 & 67 & 0.661 \\
\hline Male & 37 & 1 & \\
\hline Female & 52 & 8 & 0.157 \\
\hline Mean follow up time (months) & 21.8 & $5.63(2 \sim 15)$ & 0.064 \\
\hline Mean diameter of defect $(\mathrm{cm})$ & $3.5(1 \sim 15)$ & $394(300 \sim 600)$ & 0.000 \\
\hline Mean Size of mesh (cm) & $172(60 \sim 600)$ & $121(71 \sim 160)$ & 0.000 \\
\hline Mean OT time (min) & $75.8(30 \sim 216)$ & $3.11(1 \sim 7)$ & 0.508 \\
\hline Mean length of hospital stay (days) & $2.66(0 \sim 10)$ & $0.11(0 \sim 1)$ & 0.435 \\
\hline Mean duration of pain (months) & $0.28(0 \sim 3)$ & 0 & 0.650 \\
\hline Wound infection & $2(2 \%)$ & $1(11 \%)$ & 0.263 \\
\hline Seroma & $7(7.9 \%)$ & 0 & 0.646 \\
\hline Retention of urine & $2(2 \%)$ & 0 & 0.646 \\
\hline Recurrence & $2(2 \%)$ & $11 \%$ & \\
\hline Overall complication rate & $15 \%$ & & 0 \\
\hline
\end{tabular}


A total of 98 patients underwent laparoscopic ventral hernia repair during the defined period. Twelve (12.2\%) patients had strangulated hernia with laparoscopic repair in emergency setting. Mean diameter of hernia defect was $3.69 \mathrm{~cm}$ (range: 1 15) and mean size of mesh used was $193 \mathrm{~cm}^{2}$ (range: 60 600). Among the 98 patients, 9 of them had suprapubic hernia and 89 had non-suprapubic hernia. More than half (51\%) were incisional hernias and female (61\%). All suprapubic hernias were incisional hernias and 8 out of 9 of them were

Table 4. Types of non-midline lower abdominal hernia/extended indication of TAPE repair

\begin{tabular}{ll}
\hline 1 & Right or Left paramedian incision \\
\hline 2 & Post TRAM flap reconstruction \\
\hline 3 & Lanz or Gridiron incision \\
\hline 4 & Pfannesteil incision with significant lateral component \\
\hline
\end{tabular}
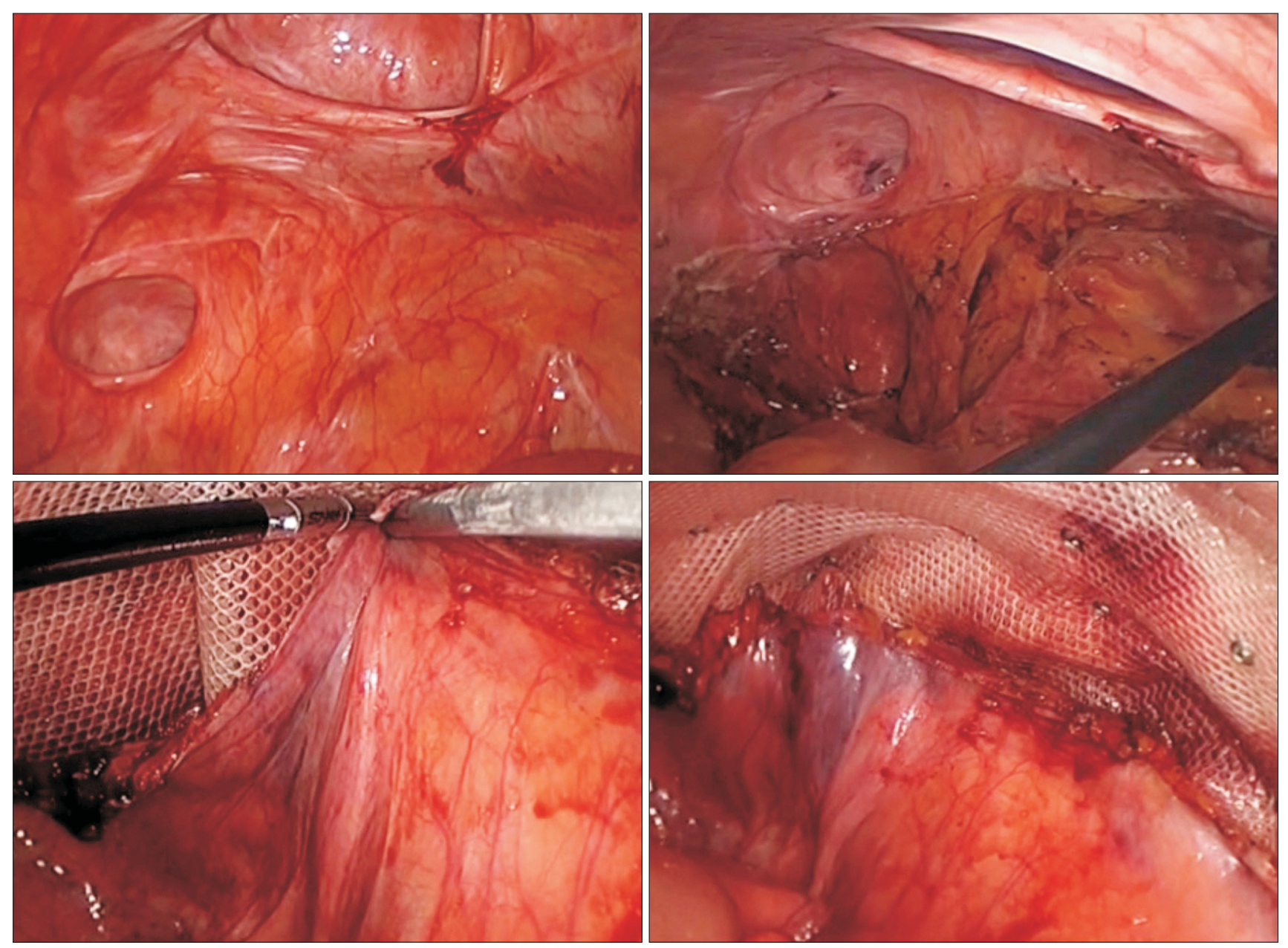

Fig. 3. Hernia after right paramedian incision, with exposure of pre-peritoneal space, and reposition of peritoneal flap and anchorage with titanium tackers with composite mesh place on pre-peritoneal space created. 
follow up.

\section{For lateral lower abdominal hernia}

Until 2016, a total of 3 lateral lower abdominal hernia requiring extended TAPE technique for the repair of incisional hernia among 123 patients with IPOM repair (Table 4). All are female patients with mean age of 49.7 (range: 41 54) years old. All patients were of ASA class II. There was 1 right lower paramedian (Fig. 3) and 2 post-TRAM flap donor site incisional hernia (Figs. 4, 5). Mean size of defect was $123.3 \mathrm{~cm}^{2}$ and mean follow-up time of 22.2 months. All patient recovered uneventfully and no recurrence noted (Table 5).

\section{DISCUSSIONS}

Mesh repair of ventral hernias is superior to primary repair especially for large hernias due to its lower recurrence rates yet similar rates of wound complication., ${ }^{3,11}$ Current evidence showed that laparoscopic mesh repair offers comparable recurrence rates and operating time, with the advantage of reduced wound infection and shorter hospital stay.,2 However, there is an increased risk of bowel injury. Suprapubic hernias are almost always incisional hernias. They are difficult to repair due to their proximity to the urinary bladder, and reduced fascial support due to the absence of the posterior rectus sheath below the arcuate line. To ensure satisfactory results of mesh repair, meshes should overlap at least $5 \mathrm{~cm}$ of the abdominal wall to allow distribution of pressure over a wider area (Pascal's principle) and permit fibrous ingrowth at the mesh-fascia interface. This principle mandates take-down of bladder in suprapubic hernia repair. Sharma et al. described 72 TAPE technique which involved bladder take-down. There was no recurrence at a mean follow-up of 4.8 years and the
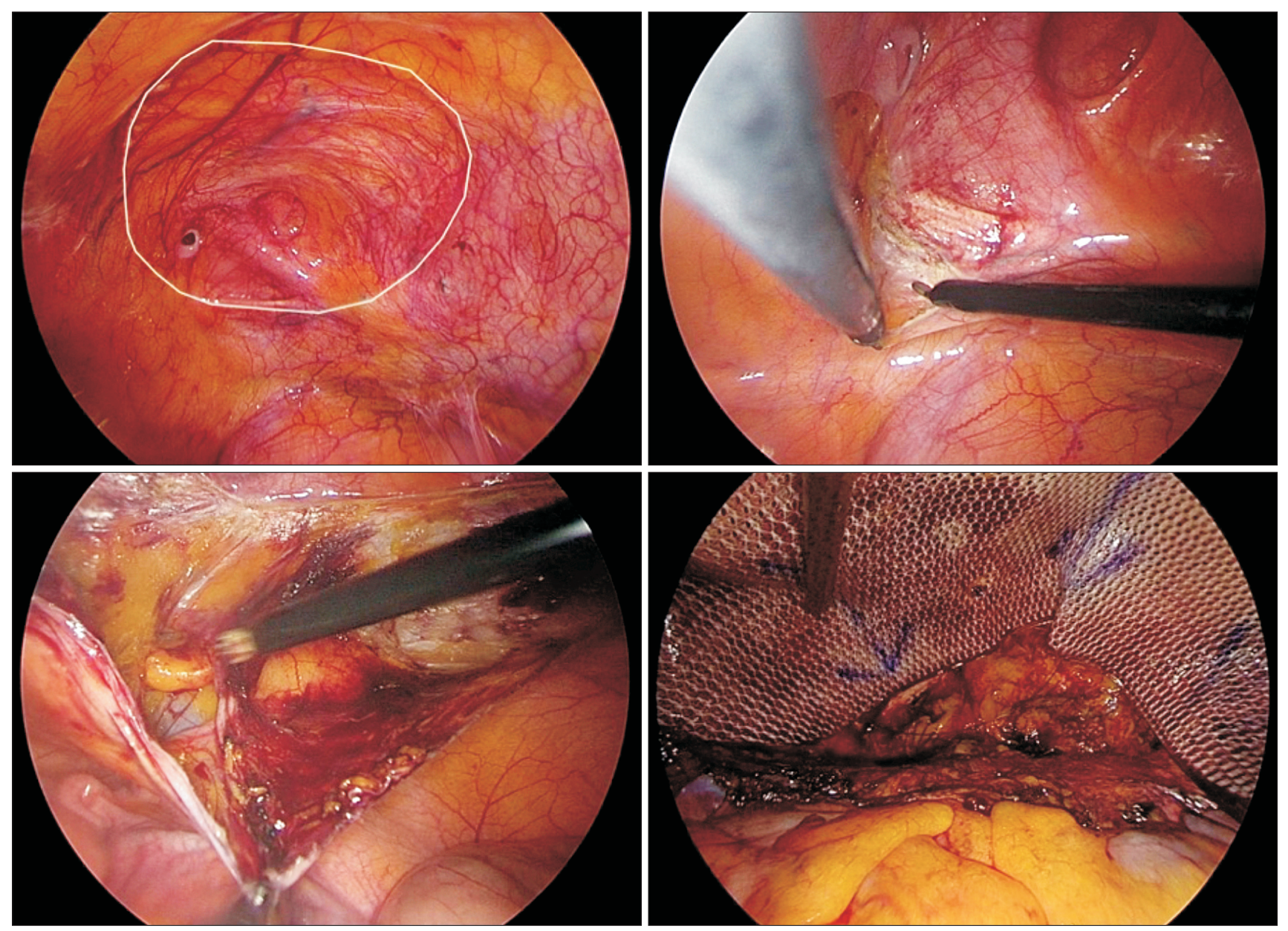

Fig. 4. Post TRAM flap donor site hernia over right lower abdomen, with peritoneal dissection started right below the edge of defect, and exposure of pre-peritoneal space over right side with round ligament and iliac vein exposed. Composite mesh anchored to the Cooper's ligament over the medial aspect. 

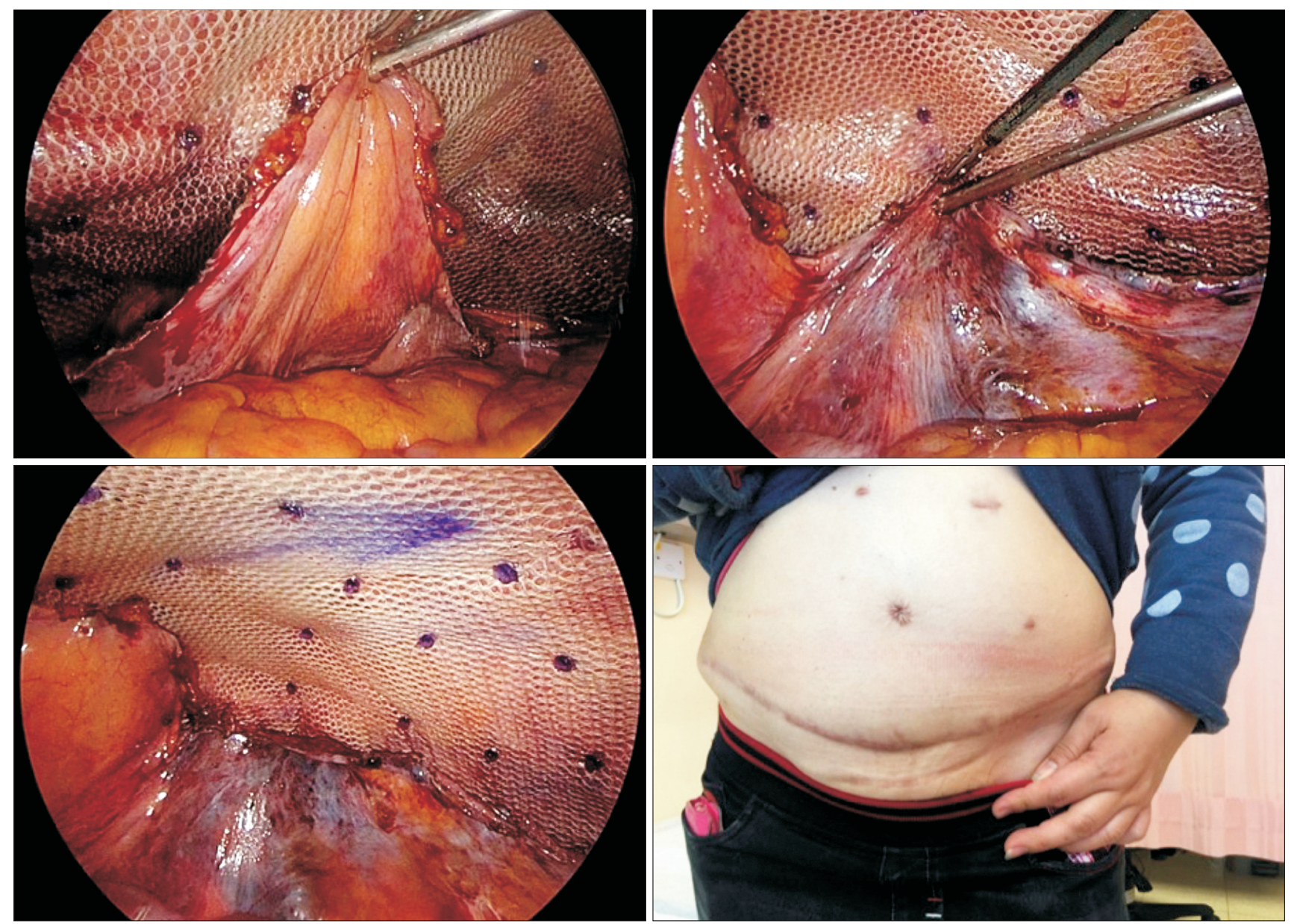

Fig. 5. Reposition and anchorage of urinary bladder at medial side. Peritoneal flap repositioned and anchored at upper border of peritoneal flap, leaving part of the composite mesh over the pre-peritoneal space created. Clinical photo 3 months post-operation.

Table 5. Patients' demographics and operative details of lateral lower abdominal hernia

\begin{tabular}{|c|c|c|c|c|}
\hline & Patient 1 & Patient 2 & Patient 3 & Mean \\
\hline Age (years old) & 54 & 41 & 54 & 49.7 \\
\hline Gender & Female & Female & Female & \\
\hline Cause of hernia & Post TRAM & Post TRAM & Right paramedian incisional hernia & \\
\hline ASA & $\|$ & $\|$ & $\|$ & \\
\hline Operation time (mins) & 114 & 161 & 143 & 139.3 mins \\
\hline Defect size (cm) & $6 \mathrm{~cm} \times 6 \mathrm{~cm}$ & $15 \mathrm{~cm} \times 15 \mathrm{~cm}$ & $4 \mathrm{~cm} \times 4 \mathrm{~cm}$ AND $3 \mathrm{~cm} \times 3 \mathrm{~cm}$ (2 defects) & $123.3 \mathrm{~cm}^{2}$ \\
\hline Mesh size $(\mathrm{cm})$ & $20 \mathrm{~cm} \times 15 \mathrm{~cm}$ & $20 \mathrm{~cm} \times 25 \mathrm{~cm}$ & $20 \mathrm{~cm} \times 25 \mathrm{~cm}$ & \\
\hline Length of stay (days) & 5 days & 5 days & 12 days & 7.33 days \\
\hline Follow up (months) & 29.4 months & 28.7 months & 8.6 months & 22.2 months \\
\hline Complication & NO & NO & NO & \\
\hline Recurrence & NO & NO & NO & \\
\hline
\end{tabular}


overall complication rate was $27.8 \%$. The complications were bleeding, ileus, urinary retention, prolonged pain or seroma which were known complications in ventral hernia surgery. There was no bladder injury. Complication rates in other literature were reported to be between 16.6 and $38 \%$ and most were minor complications only. Recurrence rates ranged from $5.5 \%$ to $6.3 \%$ at a mean follow up ranging from 2.6 to 21.1 months. ${ }^{5-7}$

\section{Hybrid pre-and intra-peritoneal mesh placement}

With the concept of IPOM repair, an overlapping of $5 \mathrm{~cm}$ of defect by prosthetic mesh is recommended for prevention of recurrence of ventral or incisional hernia - which either due to migration or shrinkage of mesh with time (which can be up to $30 \%$ decrease in mesh size). However, at some special locations, an overlapping of $5 \mathrm{~cm}$ may not be achievable due to difficulties in mesh fixation, e.g. Supra-pubic region where fixation may injury the urinary bladder or area closed to important structures such as iliac vessels, nerves where direct fixation on these areas are hazard and impossible. There is report on experience in treating this condition with open approach. ${ }^{9}$ Concept of laparoscopic TAPE technique, part of the prosthetic mesh is being placed intra-peritoneally to cover the defect, where the inferior part is being placed at preperitoneal space and covered up by the peritoneal flap after completion of procedure. And the intra-peritoneal part will be same as usual IPOM repair we are doing nowadays.

At the area where mesh being placed and fixed at preperitoneal area, the "adhesive surface is facing the abdominal wall, Cooper's ligament and pre-vesicle space, and the "nonadhesive surface will be partially covered by anterior wall of urinary bladder at area below the peritoneal incision, and part of the mesh will be placed on the peritoneal surface (intraperitoneally). This has 2 advantages: Firstly, the adhesive part will promote fibrosis and incorporation of polyester (or other material) to the pre-peritoneal structures and strengthen the repair, on the other hand, the non-adhesive surface prevents potential complication of mesh erosion to the urinary bladder. Secondly, the biomechanics within the system allows pushing the mesh to the defect via exerting the pressure first to urinary bladder then to anterior abdominal wall when patient is coughing or when intra-peritoneal pressure is increased. This "sandwiches technique promotes incorporation of mesh to the defect.

At area where fixation is impossible (e.g. lateral side at iliac vessels or nerves), the extra-peritoneal part of the mesh is placed on these important structures, then it is "fixed or $\mathrm{Cov}^{-}$ ered by peritoneal flap after mesh is placed at desired position. Then the peritoneal flap is repair and fixed at its original position as before dissection. The most inferior fixation of the mesh at the region will be at the line of peritoneal dissection, and can be safely tack along this line. The mesh inferior to this line will be protected by the peritoneal flap to prevent migration, and eventually fixation by adhesion and fibrosis induced by prosthetic mesh. Another potential way of preperitoneal fixation of the mesh is to use of either biological or synthetic glue, e.g. histoacrylic glue, which has been proven to be safe to use with low cost. We anticipate there may be small migration of mesh at these "dangerous zone at pre-peritoneal space, but hernia wont develops at these sites. Similar technique with modification has been described by Zheng et al. ${ }^{12}$ for treatment of lumbar hernia.

For midline hernia in our series, laparoscopic mesh repair in our study has an overall recurrence rate of $2 \%$, which was less than that of other published literatures. There is also in our study no recurrence for the suprapubic hernia subgroup and there is no bladder or bowel injury overall. On the other hand, suprapubic hernia repair had significantly longer operating times statistically than non-suprapubic hernia repairs (75.8 minutes vs 121 minutes, $p=0.000$ ). This was expected as extra time for bladder mobilization was necessary. However, the small difference may not have much clinical significance. The mean hospital stay was 3.11 days for suprapubic hernia repair which was comparable to non-suprapubic hernia repairs (2.66 days, $p=0.508$ ). This implied that despite longer operating time, TAPE technique for suprapubic hernia repair offers the advantage of reduced hospital stay just as the non-suprapubic subgroup. This may have implications to improved overall cost-effectiveness.

For lateral lower abdominal hernia, the reported incidence of post flap reconstruction hernia in the literature was $2.45 \%,^{13}$ 2 out of 3 in our case series were related to post TRAM surgery. Other than incisional related, there are also reports on post-traumatic, ${ }^{14,15}$ or congenital ${ }^{16}$ in origin. Despite the small number of patients included in our review for laparoscopic repair of non-midline lower abdominal hernia, however, sample size is comparable to all the literatures available nowadays with maximum number of patients included in other series on treatment of para-iliac/lower abdominal lateral hernia which included no more than 3 cases in their reports. ${ }^{9}, 10$ Our series probably is the most informative on technical details of such modification in application for this special hernia entity.

\section{CONCLUSION}

Repair of lateral lower abdominal incisional hernia with this novel modified technique is safe and feasible. A large case series of TAPE technique is required for validation of this surgical approach. 


\section{ACKNOWLEDGEMENTS}

Excellent simulated drawings by Dr. Jingsi Liu is greatly acknowledged.

\section{REFERENCES}

1) Zhang $Y$, Zhou H, Chai $Y$, Cao C, Jin $K$, Hu Z. Laparoscopic versus open incisional and ventral hernia repair: a systematic review and meta-analysis. World J Surg 2014;38:2233-2240.

2) Forbes SS, Eskicioglu C, McLeod RS, Okrainec A. Meta-analysis of randomized controlled trials comparing open and laparoscopic ventral and incisional hernia repair with mesh. Br J Surg 2009;96:851-858.

3) Burger JW, Luijendijk RW, Hop WC, Halm JA, Verdaasdonk EG, Jeekel J. Long-term follow-up of a randomized controlled trial of suture versus mesh repair of incisional hernia. Ann Surg 2004;240:578-583; discussion 583-585.

4) Mitura K, Skolimowska-Rzewuska M, Garnysz K. Outcomes of bridging versus mesh augmentation in laparoscopic repair of small and medium midline ventral hernias. Surg Endosc 2017;31:382388.

5) Carbonell AM, Kercher KW, Matthews BD, Sing RF, Cobb WS, Heniford BT. The laparoscopic repair of suprapubic ventral hernias. Surg Endosc 2005;19:174-177.

6) Palanivelu C, Rangarajan M, Parthasarathi R, Madankumar MV, Senthilkumar K. Laparoscopic repair of suprapubic incisional hernias: suturing and intraperitoneal composite mesh onlay. A retrospective study. Hernia 2008;12:251-256.

7) Varnell B, Bachman S, Quick J, Vitamvas M, Ramshaw B, Oleynikov D. Morbidity associated with laparoscopic repair of suprapubic hernias. Am J Surg 2008;196:983-987; discussion 987988.

8) Sharma A, Dey A, Khullar R, Soni V, Baijal M, Chowbey PK. Laparoscopic repair of suprapubic hernias: transabdominal partial extraperitoneal (TAPE) technique. Surg Endosc 2011;25:21472152.

9) Patel PP, Warren JA, Mansour R, Cobb WSt, Carbonell AM. A Large Single-Center Experience of Open Lateral Abdominal Wall Hernia Repairs. Am Surg 2016;82:608-612.

10) Blair LJ, Cox TC, Huntington CR, et al. Bone Anchor Fixation in Abdominal Wall Reconstruction: A Useful Adjunct in Suprapubic and Para-iliac Hernia Repair. Am Surg 2015;81:693-697.

11) Shell DHt, de la Torre J, Andrades P, Vasconez LO. Open repair of ventral incisional hernias. Surg Clin North Am 2008;88:61-83, viii.

12) Sun J, Chen X, Li J, Zhang Y, Dong F, Zheng M. Implementation of the trans-abdominal partial extra-peritoneal (TAPE) technique in laparoscopic lumbar hernia repair. BMC Surg 2015;15:118.

13) Mennie JC, Mohanna PN, O’Donoghue JM, Rainsbury R, Cromwell DA. Donor-Site Hernia Repair in Abdominal Flap Breast Reconstruction: A Population-Based Cohort Study of 7929 Patients. Plast Reconstr Surg 2015;136:1-9.

14) Cantu RV, Poka A. Traumatic abdominal hernia and lateral compression type 1 pelvic fracture: a case report. J Orthop Trauma 2006;20:289-291.

15) Nwaejike N, Pittathankal A, Walsh M. Lateral abdominal wall hernia following blunt trauma - a rare case. J Radiol Case Rep 2009;3:7-9.

16) Montes-Tapia F, Cura-Esquivel I, Gutierrez S, RodriguezBalderrama I, de la OCM. Congenital lateral abdominal wall hernia. Pediatr Int 2016;58:788-790. 